\title{
Modeling Biology in Modelica: The Human Baroreflex
}

\author{
Christopher Schölzel ${ }^{1} \quad$ Alexander Goesmann ${ }^{2} \quad$ Gernot Ernst $^{3} \quad$ Andreas Dominik $^{1}$ \\ ${ }^{1}$ KITE, Technische Hochschule Mittelhessen, Giessen, Germany, \\ \{christopher.schoelzel, andreas.dominik\}@mni.thm.de \\ ${ }^{2}$ Justus Liebig University Giessen, Giessen, Germany \\ ${ }^{3}$ Vestre Viken Hospital Trust, Kongsberg, Norway
}

\begin{abstract}
Systems biology is a field that requires complex multiscale models of systems that are evolved rather than engineered. No unifying theory exists for biology as it does for engineering domains. Thus, models appear in very diverse forms. Components can be genes, cells, organs or even whole ecosystems. These components can intuitively be represented as classes in an object-oriented language, making systems biology a perfect application for Modelica. However, we still only see very few models from this domain. In an attempt to change this, we show that Modelica can exactly reproduce the simulation results of a reference implementation of an established biological model of the human baroreflex. Our implementation highlights the strengths of Modelica like the event finding mechanism, which makes the model more precise. We also show that biological systems pose interesting challenges like signals with non-uniform delays and the interaction of complex rhythms.
\end{abstract}

Keywords: systems biology, baroreflex, cardiovascular system, heart rate variability

\section{Introduction}

Biological systems are complex, dynamic and packed with feedback loops. Even small academic examples of systems that exhibit these properties are very hard to understand and analyze for humans without proper tools (Voit, 2013, pp. 8-10). Recent support comes in form of mathematical models, forming the field of systems biology. A strong mathematical foundation can help where intuition fails and indeed there are now projects such as the Virtual Liver Network (Holzhütter et al., 2012), the Blue Brain Project (Markram, 2006) or the Physiome Project (Hunter et al., 2002) that are on the way of building comprehensive multi-scale models for complete human organs.

In all of these projects, communication between models at different scales of time and space is a key challenge. Low-level models of biochemical reactions have to be integrated into models on the cellular level which then again need to be composed together to reach the desired level of abstraction. This is made more difficult by the fact that there exists no unifying theory in biology as it does in other domains such as electrical engineering (Voit, 2013, pp.413-415). It is often not possible to build biological models as a bottom-up approach from the biochemical or genetic level, because too much is still unknown. For example, even when we narrow down the problem to these two lowest levels we still only begin to understand the role of long noncoding RNAs (Ponting et al., 2009) or glycoproteins (Ranzinger and York, 2012) in the regulation of cellular processes. Instead, researchers such as Denis Noble suggest a "middle-out" approach, that starts at the layer of abstraction where most experimental data is available (Noble, 2002).

There exist projects that aim to provide a common language for biological modeling, the most prominent being the Systems Biology Markup Language (SBML). SBML is an XML-based description format for models featuring the biochemical concept of different substances (called species) and reactions that modify the quantity of these substances. Support for the SBML is widespread in the systems biology community, but two main factors limit its usefulness for multi-scale modeling and the middleout approach. Firstly, SBML is a data format and not a programming language. Mathematical formulas have to be specified in MathML which is not a human-readable format. Therefore, often multiple tools are needed for the generation and simulation of SBML models. Secondly, SBML is specifically designed for modeling biochemical processes which enforces a bottom-up approach and makes it impossible to start the modeling process at a higher level of abstraction. Other languages that are currently used in systems biology include numerical computing environments like Matlab and Mathematica, and general purpose programming languages like $\mathrm{C}$. These languages all have sufficient expressive power and flexibility to start the modeling process at any layer of abstraction, but they also come with a lot of cognitive overhead. Scientists interested in modeling biological systems, such as physicians or biologists, have to build their equation systems and solve them by hand, or fit them 
into a specific structure for existing implementations of the desired solver. As a result, the structure of the model is fitted to the programming platform instead of the other way around. It also makes the model harder to understand and discuss both for language experts that are not familiar with the modeled system and biologists that are not familiar with the specific language constructs. Especially with large equation systems, one has to put a lot of effort into structuring his code to preserve a clear distinction between different components of the modeled systems, let alone different abstraction layers of one and the same component.

Modelica offers an elegant solution for these problems. As an object-oriented language it makes the encapsulation of subsystems into larger components intuitive and thus highly facilitates multi-scale modeling. At the highest level, a Modelica model may present cells or entire organs as components with a clear interface that hides the details of the implementation at lower levels. If desired, however, a user of the model always has the possibility to inspect a component and dig one level deeper to look at the subsystems constituting the organ or cell. In theory, this makes it possible to build arbitrarily deep nested structures without overwhelming the model user with too much detail at a single level. Additionally, the declarative acausal nature of Modelica allows to write most formulas verbatim in the same way as they may appear in the mathematical definition of the model. This greatly reduces the cognitive overhead necessary to understand, maintain and extend an existing model.

Some projects already use Modelica for tasks related to systems biology. The BioChem library allows to translate SBML models to Modelica and vice versa offering a starting point for system biologists interested in Modelica (Nilsson and Fritzson, 2005). Additionally the Physiolibrary by Mateják et al. (2014) and the HumanLib by Brunberg and Abel (2010) are both targeting the modeling of the human physiology. Yet, when we looked at three recent systems biology textbooks, we did not find any reference to Modelica (Voit, 2013; Klipp et al., 2011; Kremling, 2012), although one of these books featured a list of over 80 tools for modeling in systems biology, including Matlab, Mathematica, SBML and a variety of application-specific SBML tools (Klipp et al., 2011).

In our opinion not only system biologists can benefit from Modelica, but also the field of systems biology provides interesting challenges for Modelica modelers. In contrast to most other application areas of Modelica, biological systems are evolved rather than engineered. Specifically, this means that there is usually a high level of complicated communication between multiple parts of the system and that these interactions are not always straightforward. One major example is the the "central dogma" of molecular biology. It states that every aspect of a living system can be explained starting from the DNA which is translated to proteins. These proteins then carry out some function in the system, but do not change the genetic code. This is a natural assumption that would seem intuitive to an engineer or computer scientist: A source code defines programs regulating the behavior of a system. However, the study of epigenetics shows that there are many factors that can influence gene expression and thus change the way in which the DNA-code is read (Holliday, 2006). To make things more difficult, there is no moment in the life of an organism where a cell is constructed from nothing but DNA. Even a single fertilised egg cell still has not only inherited the DNA from its parents but also all of the other biochemical substances in this cell.

Therefore, when we build models of biological systems, we might encounter unusual connection patterns between components. These new types of problems may indicate areas, where Modelica still can be improved. As an additional argument for the study of biological models, the field of systems biology spans a large area of interesting and relevant topics, from the modeling of brain activity to finding diagnosis criteria and treatments for cardiac diseases, diabetes or cancer to the modeling of whole ecosystems like oceans (Voit, 2013, pp. 399-415). The potential of applying mathematical modeling to biological systems is vast, and with Modelica we can facilitate the generation of new insights.

With this paper we want to take a further step towards bringing together Modelica modelers and systems biologists. We show that it is not only possible to convert SBML models to Modelica with the BioChem library or build physiological models from predefined components with the Physiolibrary, but also to implement a biological model in Modelica directly from the mathematical description. As a proof of concept, we therefore implemented an established model of the human baroreflex by Seidel (1997) in Modelica and compared it with the original implementation of Seidel written in C. An introduction to the Seidel-Herzel model (SHM) will be given in section 2. The two implementations of the SHM - hereinafter called SHM-M for our Modelica version and SHM-C for Seidels C implementation - will both be described in section 3. To demonstrate that Modelica is indeed flexible enough to precisely reflect the mathematical description of the model, we directly compare the output of SHM-C and SHM-M in section 4 followed by a discussion of the results in section 5 . There we also demonstrate that biological models like the SHM fit nicely into the modeling paradigms of Modelica and highlight some interesting challenges of the implementation process. Finally, a short conclusion can be found in section 6 .

\section{The Seidel-Herzel model}

The Seidel-Herzel model (SHM) is a model of the human baroreflex that was created by Henrik Seidel and Hanspeter Herzel and first published in 1995 with the 
main purpose of analyzing heart rate variability (HRV) (Ernst, 2014). There are two main reasons why we chose this model as example for a typical biological system that can be implemented in Modelica: Firstly, although the cardiovascular system is well researched, heart diseases are still the most common cause of death worldwide (Naghavi et al., 2014). This means that models like the SHM are still relevant and may even help towards finding diagnostic criteria for heart-related diseases. The second reason to choose the SHM over other models of the human heart was that it is rather compact but still covers the effects of multiple organs. The doctorate thesis of Seidel contains the most recent version of the model, which has 24 equations and 52 parameters (Seidel, 1997).

Although there is another publication by Seidel et al. from 1998 (Seidel and Herzel, 1998), we used this version because it features several improvements of the model that were not present in the journal publication. In the following, we will give a short introduction into the components of the SHM and also explain its relevance in literature. For a more detailed explanation of the formulas, the reader is referred to Seidel's doctorate thesis (Seidel, 1997). This version of the SHM considers the physiological effects of the baroreceptors in the blood vessels, the autonomic nervous system, the lung, the sinus- and av-nodes, the heart itself and the Windkessel arteries. It does not introduce different compartments in the blood system but instead models the arterial blood pressure as a single physical quantity.

\subsection{Baroreceptors}

Baroreceptors are the sensory neurons measuring the pressure in a blood vessel. The basic neural firing frequency of the baroreceptors $v_{b}$ in the SHM is calculated with the following formula.

$$
v_{b}^{\prime}(t)=p-p_{b}^{(0)}+k_{b}^{d p} \frac{d p}{d t}
$$

This includes the effects that baroreceptors respond to the static blood pressure level $p$ as well as to an increase or decrease in blood pressure and that they only respond to blood pressure levels above a threshold $p_{b}^{(0)}$. The parameter $k_{b}^{d p}$ is a scaling factor to adjust the relative influence of the blood pressure slope.

To account for the saturation effect of baroreceptors, this value is passed through the saturation function

$$
\begin{aligned}
v_{b}(t) & =p_{b}^{c 0}\left(1+\tanh \left(\frac{v_{b}^{\prime}(t)-p_{b}^{c 0}}{p_{b}^{c 0}}\right)\right) \\
p_{b}^{c 0} & =p_{b}^{c}-p_{b}^{(0)}
\end{aligned}
$$

where $p_{b}^{c}$ is a scaling parameter to adjust the maximum of the saturation function, which lies at $2 p_{b}^{c 0}$.
In a living organism, however, the signal of baroreceptors at different parts of the body reach the autonomic nervous system (ANS) at different time instants. This effect is modeled in the SHM with a broadening function that is additionally applied to the saturated baroreceptor response.

$$
\begin{aligned}
\tilde{v}_{b}(t) & =\int_{-\infty}^{\infty} g(t-\tau) v_{b}(\tau) d \tau \\
g(t) & = \begin{cases}0 & \text { for } t \leq 0 \\
\frac{1}{\sigma} \chi_{2+\frac{\eta}{\sigma}}^{2}\left(\frac{t}{\sigma}\right) & \text { for } t>0\end{cases}
\end{aligned}
$$

In this equation $\chi_{n}^{2}$ is the probability distribution function of the chi squared distribution and $\sigma$ and $\eta$ are scaling parameters to adjust the broadening range.

\subsection{Lung}

The Lung influences the heart rate both through neural signals and the mechanical pressure in the thorax. The SHM assumes a constant breathing rate that is only modified by a noise term. The activity of respiratory neurons $v_{r}(t)$ is given by

$$
\begin{aligned}
v_{r}(t) & =\frac{1}{2}\left(1-\sin \left(2 \pi \phi_{r}(t)\right)\right) \\
\phi_{r}(t) & =\frac{t-\left(t_{r, i}-\theta_{r}\right)}{T_{r, i}}
\end{aligned}
$$

where $t_{r, i}$ is the beginning of the last inspiration phase and $\theta_{r}$ is a phase shift parameter that determines the time between the firing of respiratory neurons and the actual mechanical movement of the lungs.

The mechanical respiratory influence $f_{m}(t)$ is defined similarly by the following equation.

$$
f_{m}(t)=-\sin \left(2 \pi \phi_{r}\left(t-\theta_{r}\right)\right)
$$

Even during voluntarily controlled breathing, the breathing rate of a human is always subject to fluctuations. Seidel models these fluctuations by introducing a noise term which is applied to the mean breathing rate $\bar{T}_{r}$ at each breathing cycle with an autoregressive function.

$$
\begin{aligned}
T_{r, i} & =k_{T_{r}} \bar{T}_{r}+k_{T_{r}}^{\text {last }, 1} T_{r, i-1}+k_{T_{r}}^{\text {last }, 2} T_{r, i-2}+\sigma_{T_{r}} \xi \\
k_{T_{r}} & =\left(1-k_{T_{r}}^{\text {last }, 1}-k_{T_{r}}^{\text {last }, 2}\right)
\end{aligned}
$$

In this formula, $T_{r, i}$ is the breathing period at the $i$ th breathing cycle; $k_{T_{r}}^{\text {last, } 1}$ and $k_{T_{r}}^{\text {last }, 2}$ are parameters that determine the influence of the last and second last breathing period on the current period; and $\sigma_{T_{r}}$ is the amplitude of the white noise $\xi$. 


\subsection{Autonomic Nervous System}

The autonomic nervous system (ANS) consists of the sympathetic and the parasympathetic system. The sympathetic system increases the heartbeat frequency through the release of norepinephrine as neurotransmitter (via synapses) and as Hormone (via the blood vessels). The parasympathetic system has inhibitory influence on the heartbeat frequency through the release of the neurotransmitter acetylcholine.

The formulas for the neural activity of the sympathetic system $v_{s}$ and the parasympathetic system $v_{p}$ therefore only differ by the sign with which the baroreceptor activity enters the equation.

$$
\begin{aligned}
v_{s}^{\prime}(t) & =v_{s}^{(0)}-\left(1+k_{s}^{b r} v_{r}(t)\right) \tilde{v}_{b}(t)+k_{s}^{r} v_{r}(t) \\
v_{p}^{\prime}(t) & =v_{p}^{(0)}+\left(1+k_{p}^{b r} v_{r}(t)\right) \tilde{v}_{b}(t)+k_{p}^{r} v_{r}(t) \\
v_{s}(t) & =\max \left(0, v_{s}^{\prime}(t)\right) \\
v_{p}(t) & =\max \left(0, v_{p}^{\prime}(t)\right)
\end{aligned}
$$

Both equations have a base firing rate $v_{s / p}^{(0)}$ and scaling parameters $k_{s / p}^{r}$ for the respiratory influence and $k_{s / p}^{b r}$ for the correlation between the activity of the baroreceptors and the respiratory neurons.

\subsection{Substance Concentrations}

The SHM models several concentrations of neurotransmitters and hormones. The concentration of norepinephrine at the sinus node $\left(c_{s N e}\right)$ directly influences the pacemaker phase together with the concentration of acetylcholine $\left(c_{S A c}\right)$ at the sinus node. Additionally, norepinephrine can also act as a hormone. The ventricular concentration $c_{v N e}$ in the heart itself increases the contractility (force of the contraction). The concentration in the Windkessel arteries $c_{w N e}$ increases the stiffness of the vessel walls, resulting in a higher blood pressure during the diastole.

The release of this concentration is triggered by one neural signal and can be inhibited by another neural signal. For norepinephrine the excitatory signal comes from the sympathetic system while the parasympathetic system inhibits the release. For acetylcholine the parasympathetic system is the excitatory part and there is no inhibition modeled. Both inhibitory and excitatory signals only take effect after a delay $\theta_{c}^{x}$ and are subject to saturation. This leads us to the following equations

$$
\operatorname{ex}_{c}^{x}(t)=\operatorname{in}_{c}^{x}(t)=\tanh \left(k_{c}^{x} v_{x}\left(t-\theta_{c}^{x}\right)\right)
$$

$$
\begin{aligned}
\tau_{s N e} \frac{d c_{s N e}}{d t} & =-c_{s N e}(t)+\operatorname{ex}_{s N e}^{s}(t)\left(1-\mathrm{in}_{s N e}^{p}(t)\right) \\
\tau_{v N e} \frac{d c_{v N e}}{d t} & =-c_{v N e}(t)+\operatorname{ex}_{v N e}^{s}(t)\left(1-\mathrm{in}_{v N e}^{p}(t)\right) \\
\tau_{w N e} \frac{d c_{w N e}}{d t} & =-c_{w N e}(t)+\operatorname{ex}_{w N e}^{s}(t)\left(1-\mathrm{in}_{w N e}^{p}(t)\right) \\
\tau_{s A c} \frac{d c_{s A c}}{d t} & =-c_{s A c}(t)+\operatorname{ex}_{s A c}^{p}(t)
\end{aligned}
$$

where the $\tau_{c}$ and the $k_{c}^{x}$ are scaling parameters for the overall slope of the concentrations $c_{c}(t)$ and the influence of the inhibitory or excitatory signal.

\subsection{Sinus Node}

The sinus node is the main pacemaker of the heart. In the SHM it is modeled with the pacemaker phase $\phi(t)$ which generates a sinus signal when its value becomes one and is then directly reset to zero. The rate of the pacemaker phase increases with an increased concentration of norepinephrine and decreases with an increase in acetylcholine. The latter is additionally modified by a "phase-effectiveness curve" $F(\phi)$, because the effect of the parasympathetic signal on the pacemaker changes with the phase of the heart cycle. The resulting behavior is given by the following formula

$$
\frac{d \phi}{d t}=\frac{1}{T^{(0)}}\left(1+k_{\phi}^{s N e} c_{s N e}(t)-k_{\phi}^{s A c} c_{s A c}(t) \frac{F(\phi(t))}{\bar{F}_{\phi}}\right)
$$

$$
\begin{aligned}
\bar{F}_{\phi} & =\int_{0}^{1} F(\phi) d \phi \\
F(\phi) & =\phi^{1.3}(\phi-0.45) \frac{(1-\phi)^{3}}{(1-0.8)^{3}+(1-\phi)^{3}}
\end{aligned}
$$

where $T^{(0)}$ is the duration of the heart cycle without any input from the ANS and $k_{\phi}^{s N e}$ and $k_{\phi}^{s A c}$ are scaling parameters for the influence of the concentrations of norepinephrine and acetylcholine.

The heart period of a human is always subject to additional fluctuations that do not originate from breathing or the signals of the ANS. These influences can be implemented by replacing the parameter $T^{(0)}$ with a noisy base period $T_{n}^{(0)}$, which varies with the heartbeat number $n$ similarly to the respiratory base period in Equation 9.

$$
T_{n}^{(0)}=\bar{T}^{(0)}+k_{T^{(0)}}^{\text {last }}\left(T_{n-1}^{(0)}-\bar{T}^{(0)}\right)+\sigma_{T^{(0)}} \xi
$$

\subsection{Contraction Model}

Not every sinus signal generates a heartbeat and not every heartbeat is triggered by a sinus signal. On the one hand, if there is no sinus signal for a prolonged time period, the atrioventricular node ( $\mathrm{AV}$ node) will trigger a 
contraction by itself. This is represented by the parameter $T_{a v}$ so that a contraction is triggered if more than $T_{a v}$ seconds have passed since the last contraction at time $t_{c, n}$. On the other hand, a sinus signal does not immediately correspond to the beginning of a contraction. There is an atrioventricular concudtion delay $T_{a v c, n}$ that passes from the firing of the sinus node (which is located at the atrium) at time $t_{s, n}$ to the contraction of the ventricles. It depends on the time that has passed since the last contraction at $t_{c, n}$. Additionally, the sinus node has a refractory period $T_{\text {refrac }}$ during which no new signal may be generated. Combining these two effects, we receive the following equation for atrioventricular conduction time.

$$
T_{a v c, n}= \begin{cases}T_{a v c}^{(0)}+k_{a v c}^{t} e^{-\frac{\left(t_{s, n}-t_{c, n}\right)}{\tau_{a v c}}} & \text { if } t_{s, n}-t_{c, n}>T_{\text {refrac }} \\ \infty & \text { else }\end{cases}
$$

In this equation $T_{a v c}^{(0)}$ is the base value for the atrioventricular conduction time, $k_{a v c}^{t}$ is a scaling parameter for the influence of the time that has passed since the last contraction and $\tau_{a v c}$ is a reference value for the atrioventricular conduction time.

\subsection{Heart}

The final components of the SHM are the contraction of the heart and the Windkessel arteries that are responsible for the blood pressure increasing during the systole and decreasing during the diastole. The switch between systole and diastole is modeled explicitly by a fixed systole duration of $\tau_{\text {sys. }}$. During the systole from $t_{c, n}$ to $t_{c, n}+\tau_{\text {sys }}$ the blood pressure follows the equations

$$
\begin{aligned}
\frac{d p}{d t} & =\frac{1}{\tau_{\mathrm{sys}}} \frac{S_{n}}{C}\left(1-\phi_{\mathrm{sys}}(t)\right) e^{1-\phi_{\mathrm{sys}}(t)} \\
\phi_{\mathrm{sys}}(t) & =\frac{t-t_{c, n}}{\tau_{\mathrm{sys}}}
\end{aligned}
$$

where $C$ is a scaling constant for the contractility $S_{n}$. The value of $S_{n}$ is determined at the beginning of the systole at $t_{c, n}$ as follows.

$$
\begin{gathered}
S_{n}=S^{(0)}+\left(k_{S}^{v N e} c_{v N e}\left(t_{c, n}\right)+k_{S}^{m} f_{m}\left(t_{c, n}\right)\right) S^{\left(T_{n-1}\right)} \\
S^{\left(T_{n-1}\right)}=\left(1-\left(1-\min \left(1, \frac{t_{c, n}-t_{c, n-1}}{\hat{T}}\right)\right)^{2}\right)
\end{gathered}
$$

During the diastole, the equation for the blood pressure switches to the following formula that accounts for the effect of the Windkessel arteries. These arteries directly connected to the heart are elastic and act as a dampening system. During the systole they "store" blood by expanding the blood vessels. During the diastole they contract back to their original state slowly releasing the stored blood.

$$
\begin{aligned}
\frac{d p}{d t} & =\frac{-\left(p-p_{w}^{(0)}\right)}{\tau_{w}(t)} \\
\tau_{w}(t) & =\tau_{w}^{(0)}+k_{w}^{w N e} c_{w N e}(t)
\end{aligned}
$$

In this formula $p_{w}^{(0)}$ is the minimum blood pressure that is still present even if the Windkessel arteries are fully relaxed and the heart does not pump, $\tau_{w}^{(0)}$ is a base value for the time needed for the Windkessel arteries to fully relax, and $k_{w}^{w N e}$ is a scaling factor for the influence of the norepinephrine concentration in the arteries on this relaxation time.

\subsection{Physiological Relevance}

The SHM is able to reproduce several characteristics of complex heart rate dynamics. The first and most obvious effect are fluctuations of the heart rate with the frequency of breathing cycles called respiratory sinus arrhythmia (RSA). This behavior is not surprising as it is directly built into the model with the definition of $v_{r}$. A more interesting observation is that the model also exhibits fluctuations with a period of approximately 10 seconds, which also corresponds to a physiological phenomenon called Mayer waves (Seidel and Herzel, 1995). When investigating the reaction of the model to changes in parameter values, Seidel and Herzel (1998) also found bifurcations related to the sympathetic and parasympathetic delays, the baroreceptor sensitivity and repetitive vagal stimulation. The observed dynamical properties were in good agreement with patients with baroreceptor hypersensitivity and animal experiments. However, results by Duggento et al. (2012) show that these bifurcations can actually be triggered by most parameters of the model. They suggest that the model should be reparameterized to make all modeled variables physiologically plausible, and assume that this could lead to a " "unifying theory to account for slow oscillation' in cardiovascular variability".

Kotani et al. (2002) extended the model by noise and more detailed respiratory influences. They showed that the model can explain the synchronization between the heartbeat and the breathing frequency observed in humans (Kotani et al., 2002). In a later study they also found that the (modified) SHM could produce statistically valid simulations of congestive heart failure and primary autonomic failure - diseases that are known to affect the parasympathetic and sympathetic neural activity (Kotani et al., 2005).

To sum up, we can say that the SHM is able to produce physiologically plausible simulations of character- 
istics of both healthy patients and several disease conditions. Its potential may not have been fully exploited yet, making our implementation a possible start for future investigations.

\section{Implementations and Simulation Setup}

Thanks to Henrik Seidel we were able to use his original implementation (SHM-C) as reference for our Modelica implementation (SHM-M). It is written in C and uses a self-implemented Runge-Kutta Method for solving the differential equations. The executable allows to set starting values and parameters with a parameter file and writes the simulation result to a CSV-file.

With our Modelica-version of the Seidel-Herzelmodel we wanted to reproduce the output of SHM-C as closely as possible, while still retaining the objectoriented implementation style of Modelica. We divided the formulas of the SHM according to the physiological parts they represent to obtain small Modelica components.

On the highest level SHM-M consists of the models Baroreceptors, SinusNode, Heart, Lung, SympatheticSystem, ParasympatheticSystem, HormoneRelease and NeurotransmitterRelease as well as the compartment models BloodSystem, HormoneAmount and NeurotransmitterAmount. These models are the entry points for users of SHM-M. Therefore, we kept them as simple as possible by encapsulating the broadening and saturation of the baroreceptors, the contraction model of the heart and the phase effectiveness function into the separate classes Broaden, Tanhsaturation, Contraction, and PhaseEffectiveness. Additionally, due to the similarities in equation 11 and 12 and equations 16-19, the models sympatheticSystem and ParasympatheticSystem share a common base class ANSPart and the models HormoneRelease and NeurotransmitterRelease are even functionally equivalent to their base class SubstanceRelease only providing different icons. A diagram of the full model can be seen in Figure 1.

Most of the connections between models in the SHM$\mathrm{M}$ are implemented as a set of causal input-/outputconnectors. The neural signals and the mechanical respiratory influence as well as the boolean trigger output of the sinus node all have a clear physiological direction. The substance concentrations and the blood flow, however, are implemented using acausal connectors with flow variables. Actually this is not a mathematical requirement, because both the substance concentrations and the blood pressure are defined by a single equation in only one component that could also be implemented with causal connectors. However, physiologically the release and uptake of substances are separate processes and the blood pressure is influenced by both the Windkessel arteries and the heart itself. For a more realistic representation of these physiological properties, future versions of the model should therefore also separate these effects mathematically, which will be easier to implement using flow variables in the connectors.

With this structure, the implementation could mostly be achieved by just transferring the mathematical formulas directly to Modelica notation. Where approximations of the mathematical definition were necessary namely the broadening of the baroreceptor response with a Green's function - the same numerical algorithm used in SHM-C was implemented as a function in Modelica.

The only components where a straightforward implementation was not possible are the submodels Contraction and Broaden. The model Contraction captures the interplay of the sinus signal, refractory period and AV node and thus features rather complicated expressions in when-conditions involving discrete variables. In the current stable version of the OpenModelica compiler (Version 1.9.1) these discrete equation systems are not supported. We therefore had to implement the contraction signal using continuous variables in the when-condition as the following code snippet shows.

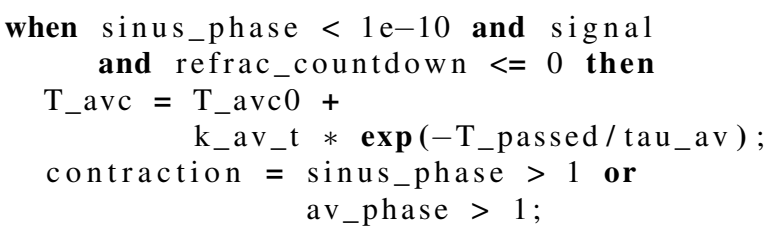

end when;

This introduced several additional phase variables for the atrioventricular conduction time, the refractory period and the period of the AV node.

The major challenge regarding the model Broad is the implementation of the convolution in Equation 4. We found no better way to calculate this convolution than to build an array with delay expressions of the baroreceptor signal with the following loop:

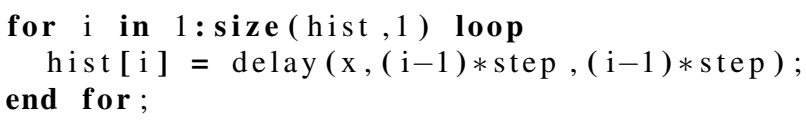

This implementation works as desired for small broadening lengths, but becomes extremely slow for larger values.

As a final difference between SHM-M and SHM-C we did not implement the noise model for the breathing frequency and the heartbeat duration, because this noise would only complicate the comparison of the two models. Instead, to obtain comparable data, noise was also disabled in SHM-C through parameter settings. 


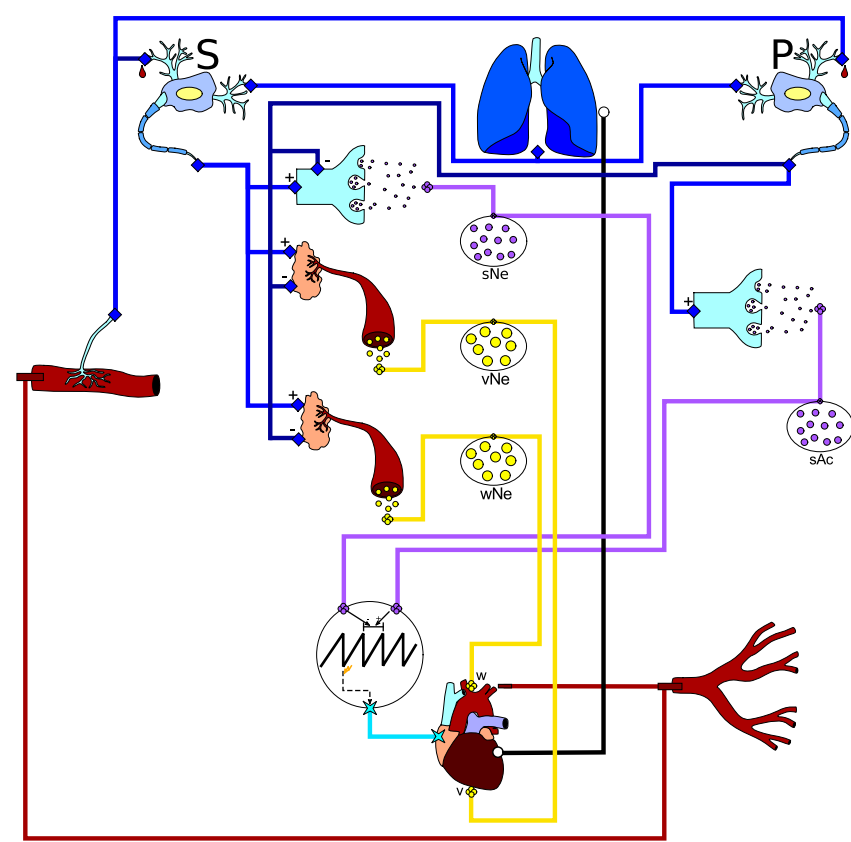

Figure 1. Diagram view of the SHM-M implementation showing the components of the model.

The simulation of our model was performed using OpenModelica. The Runge-Kutta method implemented in OpenModelica uses the same classical RK4Parameters that are also used in SHM-C. Therefore we only had to use the same step size and the same set of starting values and parameters for the simulation to obtain directly comparable results.

We used the standard parameter set by Seidel and only adjusted the manually defined starting values in SHM-C to match the starting values of SHM-M that were calculated by OpenModelica. The resulting parameter configuration can be seen in Table 1 . We then did a simulation with both models for 1000 seconds with a step size of one millisecond and recoded the important values in steps of ten milliseconds as well as the duration and end time for each heartbeat.

\section{Results}

The SHM was designed for the analysis of heart rate variability. Therefore, to compare different implementations it is most important to look at the duration of heartbeats and the blood pressure. A direct comparison of the time series for these physical quantities can be seen in Figure 2. For the blood pressure both curves have no visual differences until 5 seconds after the start of the simulation, when SHM-M starts to run ahead slightly. At the end of the simulation, the situation is similar: The only difference between the curves seems to be a time shift. For the heartbeat duration the differences are already noticeable at the first heart beat, which is 3 milliseconds longer in SHM-M than in SHM-C.

To better quantify these differences, we plotted the
Table 1. Parameter and initial values used for the comparison of SHM-C and SHM-M.

\begin{tabular}{|c|c|c|c|}
\hline Parameter & Value & Parameter & Value \\
\hline \multicolumn{2}{|c|}{ Baroreceptors } & \multicolumn{2}{|l|}{ Sinus node } \\
\hline$p_{b}^{(0)}$ & 60 & $T^{(0)}$ & 0.9 \\
\hline$k_{b}^{d p}$ & 0.06 & $k_{\phi}^{s N e}$ & 0.6 \\
\hline$p_{b}^{(c)}$ & 120 & $k_{\phi}^{s A c}$ & 0.2 \\
\hline$\sigma$ & 0.001 & \multicolumn{2}{|l|}{ Contraction } \\
\hline$\eta$ & 0.01 & $T_{a v c}^{(0)}$ & 0.09 \\
\hline Lung & & $k_{a v c}^{t}$ & 0.78 \\
\hline$\theta_{r}^{(0)}$ & 0.16 & $\tau_{a v c}$ & 0.11 \\
\hline $\bar{T}_{r}$ & 4 & $T_{\text {refrac }}$ & 0.22 \\
\hline$k_{T_{r}}^{\text {last }, 1}$ & 0 & $T_{a v}$ & 1.7 \\
\hline$k_{T_{r}}^{\text {last }, 2}$ & 0 & \multicolumn{2}{|l|}{ Heart } \\
\hline$\sigma_{T_{r}}$ & 0 & $\tau_{\mathrm{sys}}$ & 0.125 \\
\hline ANS & & $C$ & 2 \\
\hline$v_{s}^{(0)}$ & 50 & $S^{(0)}$ & 110 \\
\hline$k_{s}^{b r}$ & 0.38 & $k_{S}^{v N e}$ & 110 \\
\hline$v_{s}^{r}$ & 30 & $k_{S}^{m}$ & 0 \\
\hline$v_{p}^{(0)}$ & 10 & $p_{w}^{(0)}$ & 0 \\
\hline$k_{p}^{b r}$ & 0.38 & $\tau_{w}^{(0)}$ & 1.3 \\
\hline$v_{p}^{r}$ & 30 & $k_{w}^{r N e}$ & 0.8 \\
\hline \multicolumn{2}{|c|}{ Concentrations } & $\hat{T}$ & 1 \\
\hline$k_{s N e}^{s}$ & 0.014 & \multicolumn{2}{|l|}{ Initialization } \\
\hline$\theta_{s N e}^{s}$ & 2 & $p(0)$ & 100 \\
\hline$k_{s N e}^{p}$ & 0.006 & $c_{s N e}(0)$ & 0.12 \\
\hline$\theta_{s N e}^{p}$ & 0.4 & $c_{s A c}(0)$ & 0.5 \\
\hline$\tau_{s N e}$ & 2 & $c_{v N e}(0)$ & 0.12 \\
\hline$k_{v N e}^{s}$ & 0.014 & $c_{r N e}(0)$ & 0.12 \\
\hline$\theta_{v N e}^{s}$ & 2 & $T_{0}$ & 1 \\
\hline$k_{v N e}^{p}$ & 0.006 & $t_{c, 0}$ & -1 \\
\hline$\theta_{v N e}^{p}$ & 0.4 & $T_{a v c}(0)$ & 0.15 \\
\hline$\tau_{v N e}$ & 4 & $S_{0}$ & 110 \\
\hline$k_{r N e}^{s}$ & 0.014 & & \\
\hline$\theta_{r N e}^{s}$ & 3 & & \\
\hline$k_{r N e}^{p}$ & 0 & & \\
\hline$\theta_{r N e}^{p}$ & 0.4 & & \\
\hline$\tau_{r N e}$ & 4 & & \\
\hline$k_{S A c}^{p}$ & 0.005 & & \\
\hline$\theta_{s A c}^{p}$ & 0.4 & & \\
\hline$\tau_{s A c}$ & 0.05 & & \\
\hline
\end{tabular}



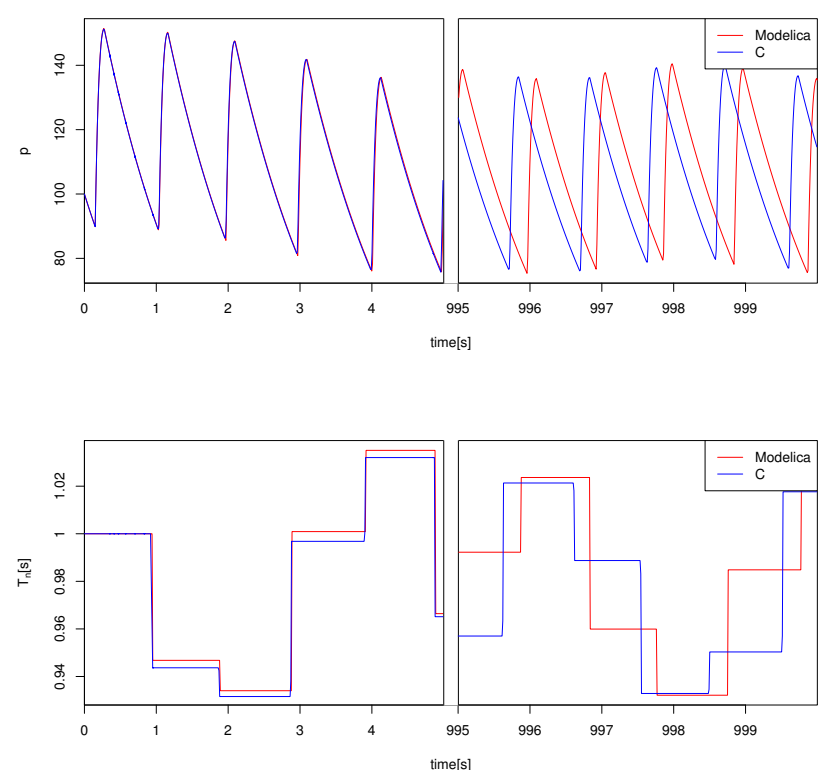

Figure 2. Comparison of time series of blood pressure $p$ and heartbeat duration $T_{n}$ between SHM-C (blue) and SHM-M (red) for a simulation time of 1000 seconds, showing seconds 0 to 5 and seconds 995 to 1000 .

difference between heartbeat durations in SHM-M and SHM-C against the standard deviation between heartbeat durations in SHM-C. The result can be seen in Figure 3. It turns out that the duration of the first 40 heartbeats only differs by less than 10 milliseconds with a standard deviation of 34 milliseconds. The plot also shows that on average SHM-M produces heartbeat periods that are 3 milliseconds longer.

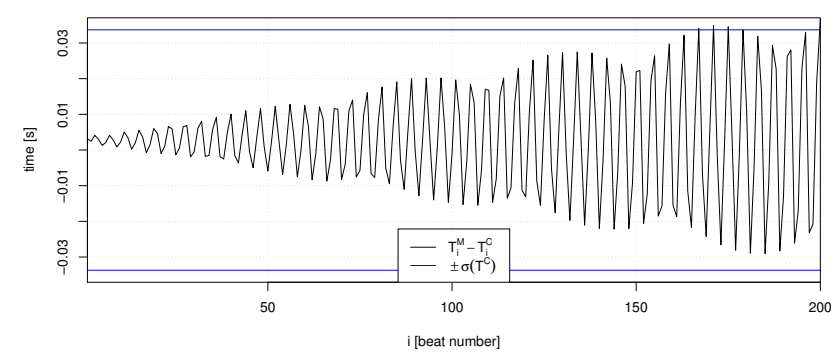

Figure 3. Difference between RR-Intervals of SHM-M and SHM-C relative to the standard deviation of RR-Intervals in SHM-C. Values above zero represent RR-Intervals that are longer in SHM-M compared to SHM-C.

While absolute differences can give an impression of the size of possible calculation errors, for the SHM it is much more interesting to look at the long-time behavior of the model. Seidel used a plot of the spectral density of RR-Intervals (i.e. heartbeat durations) as one of his main arguments for the physiological plausibility of his model. We therefore also compared SHM-C and SHM-M on the frequency domain. The result can be seen in Figure 4. The plot shows a clear peak identical in magnitude and position at approximately $0.25 \mathrm{~Hz}$, which corresponds to the breathing frequency and can thus be thought to represent respiratory sinus arrhythmia. We can also see another less pronounced peak for both implementations at approximately $0.1 \mathrm{~Hz}$ which Seidel attributes to Mayer waves. However, in SHM-M the peak at $0.25 \mathrm{~Hz}$ is much sharper than in SHM-C.
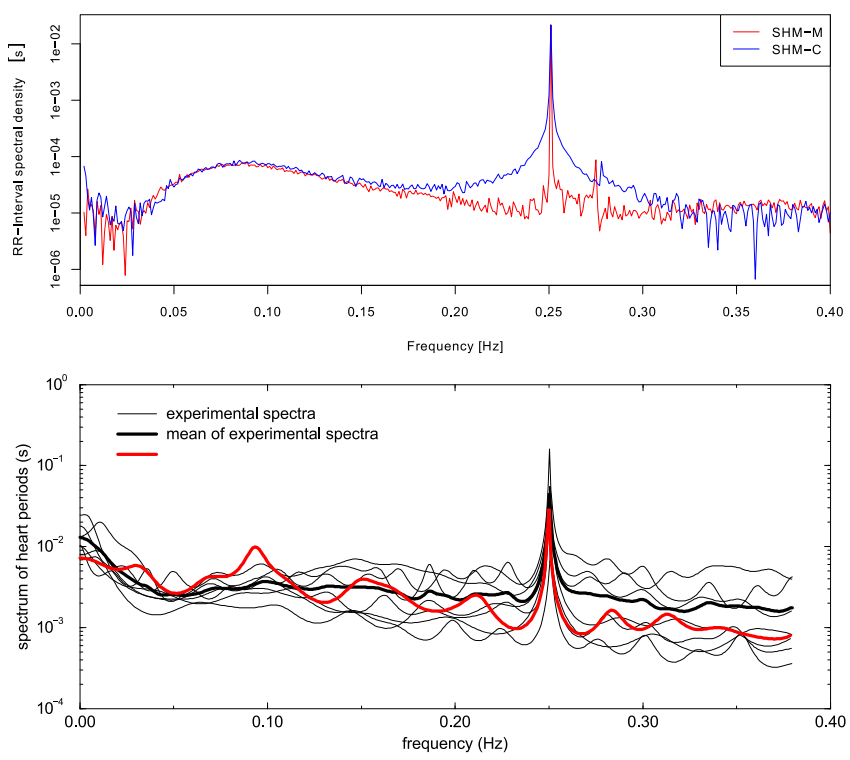

Figure 4. Top: Comparison of spectral density of RR-Intervals in SHM-C (blue) and SHM-M (red) after a simulation for 1000 seconds with standard parameters. Bottom: Figure from Seidel's doctorate thesis comparing spectral density of RR-Intervals of SHM with noise (red) and experimental data (black) (Seidel, 1997).

\section{Discussion}

The comparison between both implementations SHM-M and SHM-C shows that our proof of concept was successful. At the beginning of the simulation, the blood pressure stays almost the same. There are noticeable differences in the heartbeat duration, but these are not unexpected as the event finding mechanism of Modelica can determine the exact time at which an event occurs more precisely compared to the simple check after each Runge-Kutta step implemented in SHM-C. This is also consistent with the sharper peak in the frequency domain observed in Figure 4. Experimental data with voluntarily controlled breathing actually shows a rather smooth RSA-related peak in the frequency domain similar to SHM-C. However, the reason for this is that the subjects naturally cannot time their breathing to the exact millisecond, introducing a noise to the breathing frequency. This type of noise has been incorporated into the model by Seidel and it can also be incorporated in SHM-M. Our model therefore may allow a more precise 
analysis of the theoretical effects of RSA without sacrificing realism.

Now that we have seen that SHM-M is able to reproduce the simulation results of SHM-C we can ask what makes the new implementation (or biological models in general) interesting from a Modelica perspective. First of all, the notion of a system composed of multiple organs fits nicely into the object-oriented paradigm and leads to a natural and intuitive class structure. In fact, each subsection in the explanation of the SHM in section 2 corresponds exactly to a Modelica model in SHM-M. The model structure directly reflects the structure of the real world system and thus makes the model very explainable and accessible for domain experts. Furthermore, encapsulation, inheritance and the reuse of objects instantiated with different parameters could be applied to yield a hierarchical structure that hides implementation complexities and avoids code repetition. The idea of hierarchically structured taxonomies is deeply rooted in biology. It therefore seems reasonable to expect that most biological models can be implemented in such a clean and intuitive manner using Modelicas object-oriented approach.

Additionally, due to the tendency of biological systems to feature multiple complex rhythms, these models can showcase the strength of Modelica's event finding mechanism in comparison to a naive implementation of the Runge-Kutta- or Euler-method or other tools that mainly focus on continuous modeling.

These complex rhythms also turned out to be one of the two major challenges that arose during the implementation of SHM-M. As already mentioned a straightforward implementation of the contraction model was not possible in OpenModelica, because the nontrivial conditions in the when-equations formed a discrete equation system. We did not test the model with other compilers, so this may be only an issue with OpenModelica, but nonetheless our biological model requires a feature that seems not as crucial for most other application areas of Modelica.

The second major implementation challenge was the broadening function used for the baroreceptors. To assess the performance issues with the implementation using direct delay equations, we recorded the time taken for a simulation over 1000 seconds for both SHM-M and SHM-C with broadening lengths ranging from $0.1 \mathrm{sec}-$ onds to 3 seconds. We found that simulation times of SHM-M rise linearly from 75 seconds with a broadening length of 0.1 seconds to as much as one hour for a broadening length of 3 seconds. In contrast, SHM-C only shows an increase from 16.6 to 21.3 seconds respectively. This results suggest that OpenModelica uses a separate history buffer for each delay equation in the loop. If this is the case, an increase of the broadening length by only one simulation step would require the allocation and management of an additional buffer of the same size as the single history buffer used in SHM-C, explaining the additional overhead. We are not aware of a language construct that allows to indicate that the delay equations in the loop may share the same buffer. Building the buffer manually in Modelica is also not possible, because the language itself has no notion of discrete simulation steps. This performance issue is therefore hard to fix as a Modelica programmer and would possibly be an argument to include convolutions as a language element.

We can therefore conclude that the SHM, as a model that exhibits the typical properties of biological models in general, does not only fit nicely into the modeling style of Modelica but also has some challenging aspects that point to possible areas of improvement for OpenModelica or Modelica in general. This suggests that biological models could indeed become a new and interesting application area for Modelica.

\section{Conclusion}

With our implementation of the SHM we demonstrated as a proof of concept that Modelica is perfectly suited for the implementation of biological systems in a natural representation. The language can directly reflect the biological composition of the system instead of having to fit the system into the language constructs. This is shown by the fact that our Modelica version of the SHM - which uses a lot of the features of Modelica such as acausal declarations, encapsulation and component reuse through instantiation and inheritance - can reproduce the same behavior as the original reference implementation in C. Moreover, it is in some parts even more precise thanks to the event finding mechanisms of Modelica.

We also demonstrated that new challenges that are not present in other domains may arise when we model evolved rather than engineered systems. The interaction of complex rhythms together with time-varying signal conduction delays lead to a contraction model that could not be intuitively implemented with the current version of the OpenModelica compiler. Additionally, implementing the behavior that the baroreceptor signal reaches the ANS through many different nervous connections with varying delays required a convolution that seems to be a performance bottleneck.

These are strong arguments both for biologists to choose Modelica over a general purpose programming language and for Modelica modelers to look for interesting applications and models in the systems biology domain. This paper laid the ground for the implementation of more biological models from the side of the Modelica community, but to encourage interdisciplinary research we also have to take the opposite perspective. We need to investigate the benefits of Modelica more closely in regard to the needs of systems biologists. A first step could be to reparameterize the SHM as suggested by Duggento et al. (2012) and to incorporate additional components that can simulate vagal stimulation and different disease conditions (which is possible but 
cumbersome with the $\mathrm{C}$ implementation). We also plan to extend the SHM to a multi-scale model, for example by exchanging the heart model with a more detailed representation modeling individual heart cells. Finally, it would be interesting to embed the model into the Physiolibrary to provide a single point-of-entry for biologists and physicians interested in physiological modeling with Modelica. We believe that there is a lot of potential for interesting projects involving biological models in Modelica and we are looking forward to seeing more of them in the future.

\section{References}

Anja Brunberg and Dirk Abel. Simulation verkoppelter physiologischer Regelkreise mit Hilfe der objektorientierten Modellbibliothek „HumanLib“. In Automatisierungstechnische Verfahren für die Medizin, 9. Workshop, Tagungsband, pages 29-30, Zürich, Switzerland, 2010. doi:10.1524/auto.2011.0951.

Andrea Duggento, Nicola Toschi, and Maria Guerrisi. Modeling of human baroreflex: Considerations on the Seidel-Herzel model. Fluctuation and Noise Letters, 11(1): 1240017, 2012. doi:10.1142/S0219477512400172.

Gernot Ernst. Heart rate variability. Springer-Verlag, London, England, 2014. ISBN 978-1-4471-4308-6. doi:10.1007/978-1-4471-4309-3.

Robin Holliday. Epigenetics: A historical overview. Epigenetics, 1(2):76-80, 2006. doi:10.4161/epi.1.2.2762.

Hermann-Georg Holzhütter, Dirk Drasdo, Tobias Preusser, Jörg Lippert, and Adriano M. Henney. The virtual liver: A multidisciplinary, multilevel challenge for systems biology. Wiley Interdisciplinary Reviews: Systems Biology and Medicine, 4(3):221-235, 2012. doi:10.1002/wsbm.1158.

Peter Hunter, Peter Robbins, and Denis Noble. The IUPS human physiome project. Pflügers Archiv - European Journal of Physiology, 445(1):1-9, 2002. doi:10.1007/s00424-0020890-1.

Edda Klipp, Wolfram Liebermeister, Christoph Wierling, Axel Kowald, Hans Lehrach, and Ralf Herwig. Systems biology: A textbook. Wiley-Blackwell, Hoboken, New Jersey, 2011.

Kiyoshi Kotani, Kiyoshi Takamasu, Yosef Ashkenazy, H. Stanley, and Yoshiharu Yamamoto. Model for cardiorespiratory synchronization in humans. Physical Review E, 65(5): 051923, 2002. doi:10.1103/PhysRevE.65.051923.

Kiyoshi Kotani, Zbigniew Struzik, Kiyoshi Takamasu, H. Stanley, and Yoshiharu Yamamoto. Model for complex heart rate dynamics in health and diseases. Physical Review E, 72(4):041904, 2005. doi:10.1103/PhysRevE.72.041904.

Andreas Kremling. Kompendium Systembiologie: Mathematische Modellierung und Modellanalyse. Vieweg+Teubner, Wiesbaden, Germany, 2012. ISBN 978-3-8348-1907-9.

Henry Markram. The blue brain project. Nature Reviews Neuroscience, 7(2):153-160, 2006. doi:10.1038/nrn1848.
Marek Mateják, Tomáš Kulhánek, Jan Šilar, Pavol Privitzer, Filip Ježek, and Jiří Kofránek. Physiolibrary - Modelica library for physiology. In Proceedings of the 10th International Modelica Conference, pages 499-505, Lund, Sweden, 2014. doi:10.3384/ecp14096499.

Mohsen Naghavi, Haidong Wang, Rafael Lozano, Adrian Davis, Xiaofeng Liang, Maigeng Zhou, and Stein Emil Vollset. Global, regional, and national age-sex specific allcause and cause-specific mortality for 240 causes of death, 1990-2013: a systematic analysis for the Global Burden of Disease Study 2013. The Lancet, 385(9963):117-171, 2014. doi:10.1016/S0140-6736(14)61682-2.

Emma Larsdotter Nilsson and Peter Fritzson. A metabolic specialization of a general purpose modelica library for biological and biochemical systems. In Proceedings of the 4th International Modelica Conference, pages 85-93, Hamburg, Germany, 2005.

Denis Noble. The rise of computational biology. Nature Reviews Molecular Cell Biology, 3(6):459-463, 2002. doi:10.1038/nrm810.

Chris P. Ponting, Peter L. Oliver, and Wolf Reik. Evolution and functions of long noncoding RNAs. Cell, 136(4):629-641, 2009. doi:10.1016/j.cell.2009.02.006.

R. Ranzinger and William S. York. Glyco-bioinformatics today (august 2011) - solutions and problems. In Proceedings of the 2nd Beilstein Symposium on Glyco-Bioinformatics, Potsdam, Germany, 2012.

Henrik Seidel. Nonlinear dynamics of physiological rhythms. $\mathrm{PhD}$ thesis, Technische Universität Berlin, Berlin, Germany, 1997.

Henrik Seidel and Hanspeter Herzel. Modelling heart rate variability due to respiration and baroreflex. In Erik Mosekilde and Ole G. Mouritsen, editors, Modelling the Dynamics of Biological Systems, number 65 in Springer Series in Synergetics, pages 205-229. Springer, Berlin Heidelberg, Germany, 1995. ISBN 978-3-642-79292-2.

Henrik Seidel and Hanspeter Herzel. Bifurcations in a nonlinear model of the baroreceptor-cardiac reflex. Physica D: Nonlinear Phenomena, 115(1-2):145-160, 1998. doi:10.1016/S0167-2789(97)00229-7.

Eberhard O. Voit. A first course in systems biology. Garland Science, New York City, New York, 2013. ISBN 978-08153-4467-4 